\title{
QUENCH ENERGIES OF POTTED MAGNETS, II*
}

\author{
Lawrence Dresner \\ Oak Ridge National Laboratory \\ Oak Ridge, Tennessee
}

DE85 016962

\begin{abstract}
Several different formulas have been proposed for estimating quench energies of po\%ted magnets ${ }^{1-3}$ These formulas, although based on plausible approximations, disagree with one another by faciors of 2 to 6. By the use of maximum and minimum principles for the heat balance equation, it has been possible to determine whether each estimate is an upper or lower boind and to show that some estimates majorize others.
\end{abstract}

\section{INTRODUCTION}

By "quench energy" of a magnet, we mean the largest, instantaneous energy deposit at $a$ point from which the magnet can still recover. In two earlier papers, ${ }^{1,2}$ I applied a combination of dimensional and group-theoretic arguments to the time-dependent heat balance equation and found without detaili-d calculation a formula for the quench energies of potted magnets complete up to a single undetermined constant. In these earlier papers, I considered two extreme models of the winding, namely, (1) treating it as an anisotropic three-dimensional (3-D) continuum and (2) ignoring heat conduction in the epoxy and treating it as a one-dimensional (1-D) continuum. For each model, I proposed approximate solutions to the time-dependent heat balance equation in order to estimate the undetermined constant. Wilson proposed a different approximate method ${ }^{3}$ of estimating the undetermined constant based on consideration of the steady-state heat balance equation. These estimates, although based on plausible approximations, disagree with one another by factors of 2 to 6 . In this paper, 1 show by using maximum and minimum principles for the heat balance equation, ${ }^{4}$ how to determine whether each estimate is an upper or lower bound and how to show that some estimates majorize others.

This paper is a direct follow-on to ref. 2, and to best appreciate it, the reader should be familiar with the material in ref. 2 . For this reason, and because the editor's demand for brevity makes it impossible for me to write this paper in a self-contained way by repeating certain parts of ref. 2 , I shall write it as though ref. 2 lay on the table before the reader. In what follows, the notation (2.4) will mean Eq. (4) of ref. 2, while the notation

'Research sponsored by the Office of Fusion Energy, U.S. Department of Energy, under Contract No. DE-AC05-84OR21400 with Martin Marietta Energy Systems, Inc. 
(4) will mean Eq. (4) of the present paper. The symbols are the same as those used in ref. 2 .

\section{ANISOTROPIC 3-D CONTINUUM}

As we see in Sects. 2.2 and 2.3, the mathematicel task we face in determining the quench energies of potted magnets is solving the following nonlinear eigenvalue problem. The infinite-medium solutions of the heat balance equation,

$$
\frac{\partial \theta}{\partial \tau}=\frac{1}{\xi^{2}} \frac{\partial}{\partial \xi}\left(\xi^{2} \frac{\partial \theta}{\partial \xi}\right)+g(\theta)
$$

and

$$
q(\theta)=\left\{\begin{array}{ll}
0 & 0<\theta<1-i \\
(\theta+i-1) / i & 1-i<\theta
\end{array},\right.
$$

with the initial condition

$$
\theta=q \frac{\exp \left(-\xi^{2} / 4 \tau\right)}{(4 \pi \tau)^{3 / 2}} \quad(\text { very small } \tau)
$$

$\left(\xi^{2}=\eta^{2}+\zeta^{2}\right.$ is the square of the radius vector), lead either to recovery $(\lim \theta=0$ for all $\xi$ ) or to a quench ( $\lim _{\rightarrow \infty} \theta=\infty$ for all $\xi$ ) according to the magnitude of $q$. What is the limiting value of $q$ that separates recovery from quench?

The limiting value of $q$ can be at most a function of $i$, and the group-tineoretic arguments of Sect. 2.3 show it to depend on $i$ according to $q=C(1-i) i^{3 / 2}$, where $C$ is a constant rot determined by the group-theoretic argument. To estimate $C$, I used in ref. 2 an approximate solution having the form of Eq. (2.5) but in which $q$ was taken to depend on $\tau$; that is, $q=H(\tau)$. Such a solution is our jumping-off point here.

If we substitute Eq. (2.5) into Eq. (2.3a), we get

$$
\frac{1}{\xi^{2}} \frac{\partial}{\partial \xi}\left(\xi^{2} \frac{\partial \theta}{\partial \xi}\right)+g(\theta)-\frac{\partial \theta}{\partial \tau}=g\left[H \frac{\exp \left(-\xi^{2} / 4 \tau\right)}{(4 \pi \tau)^{3 / 2}}\right]-\frac{\exp \left(-\xi^{2} / 4 \tau\right)}{(4 \pi \tau)^{3 / 2}} \dot{H}
$$

where $\dot{H}$ is an abbreviation for $d H / d \tau$. In ref. 2, Sect 2.4 , the function $H$ was determined by requiring the right-hand side (RHS) of Eq. (1) to vanish at $\xi=0$. Then

$$
\operatorname{RHS}(1)=g\left[H \frac{\exp \left(-\xi^{2} / 4 \tau\right)}{(4 \pi \tau)^{3 / 2}}\right]-\exp \left(-\xi^{2} / 4 \tau\right) g\left[\frac{H}{(4 \pi \tau)^{3 / 2}}\right]
$$

Now if the function $g$ is concave upwards [as in Eq. (2.8)], then the RHS of Eq. (2) is $<0$. From this it follows that for the ansatz of Eq. (2.5) $\frac{1}{\xi^{2}} \frac{\partial}{\partial \xi}\left|\xi \frac{\partial \theta}{\partial \xi}\right|+g(\theta)<\partial \theta / \partial \tau$.

Suppose now that $u$ is the exact solution of Eqs. (2.3a) and (2.8), having Eq. (2.5) with $\mathrm{q}=H(0)$ as initial condition. If we define $w=(\theta-u) e^{-\gamma \tau}$, where $\gamma$ is a constant with a value to be given later. then using the law of the mean we find that $w$ satisfies 


$$
\frac{1}{\xi^{2}} \frac{\partial}{\partial \xi}\left(\xi^{2} \frac{\partial w}{\partial \xi}\right)+\{\dot{g}[k \theta+(1-k) u]-\gamma\} w<\frac{\partial w}{\partial \tau} \quad(0<k<1)
$$

with the boundary, initial, and symmetry conditions being

$$
w(\infty, \tau)=0, w(\xi, 0)=0, \text { and } \frac{\partial w}{\partial \xi}(0, \tau)=0
$$

If we choose $\gamma>\max (\dot{g})$, we can prove (see the appendix) that $w>0$, that is, that $\theta>u$.

If $H(0)$ is chosen so that $\theta$ recovers, then $u$ must also recover. In ref. 2 , it was shown that if $H(0)=C(1-i) i^{3 / 2}$ with $C=6 \pi^{2}$, then $\theta \leqslant 1-i$ for large enough $\tau$. Thus, ultimately $\alpha<1-i$ for large enough $\tau$. Because there is no Joule power production for $u<$ $1-i, u$ must finally approach zero as the result of ordinary heat diffusion. Therefore, $q_{\delta}$, the largest value of $q$ for which $u$ still recovers, must be $\geqslant 6 \pi^{2}(1-i) i^{3 / 2}$. This last result means that Eq. (2.19) gives a lower bound to $Q$, the quench energy, in the anisotropic 3-D continuum model.

\section{WILSON'S METHOD OF DETERMINING $C$}

Wilson proposed ${ }^{3}$ a way of estimating the constant $C$ using the steady-state solutions $v(\xi)$ of the heat balance equation that cbey the natural boundary condition $(\partial v / \partial \xi)_{\xi=0}=$ 0 and the artificial boundary condition $v(R)=0$. Wilson calculates the the energy of formation of these steady states, $\int_{0}^{\infty} v(\xi) 4 \pi \xi^{2} d \xi$, and finds its minimum with respect to the parameter $R$. This he takes to be his estimate of the quench energy.

The group-theoretic argument of Sect. 2.3 shows that this estimate, too, must have the form $C(I-i) i^{3 / 2}$, and a lengthy but straightforward computation gives the value $C=$ 356.4 .

How is this estimate related to the true value of the quench energy? To answer this question, we take $R$ to be fixed and study the time-dependent solutions of Eqs. (2.3a) and (2.8), which have multiples of $v(\xi)$ as their initial condition: $\theta(\xi, 0)=(1+\epsilon) v(\xi)$, where $\epsilon$ is arbitrary. If tre substitute $\theta(\xi, 0)=(1+\epsilon) v(\xi)$ into Eq. $(2.3 a)$, we find

$$
\frac{1}{\xi^{2}} \frac{\partial}{\partial \xi}\left[\xi^{2} \frac{\partial \theta(\xi, 0)}{\partial \xi}\right]+g[\theta(\xi, 0)]=g[(1+\epsilon) v]-(1+\epsilon) g(v)\left\{\begin{array}{ll}
>0, & \epsilon<0 \\
<0, & \epsilon>0
\end{array},\right.
$$

due to the function $g$ being concave upwards. If we now set $w=[\theta(\xi, \tau)-\theta(\xi, 0)] e^{-\gamma \tau}$, we find by subtracting Eq. (4) from Eq. (2.3a) that

$$
\frac{1}{\xi^{2}}-\frac{\partial}{\partial \xi}\left\{\xi^{2} \frac{\partial w}{\partial \xi}\right]+\{\dot{g}[k \theta(\xi, \tau)+(1-k) \theta(\xi, 0)]-\gamma\} w\left\{\begin{array}{ll}
<\partial w / \partial \tau, & \epsilon>0 \\
>\partial w / \partial \tau, & \epsilon<0
\end{array}(0<k<1) .\right.
$$

Furthermore, $w$ obeys the boundary, initial, and symnistry ccnditions of Eq. (3b). Again we choose $\gamma>\max (\dot{\mathrm{g}})$; then we prove exaivly as before (see Appendix) that if $\epsilon>0$, then $w>0$; that is, $\theta(\xi, \tau)>\theta(\xi, 0)$. But this means the solution $\theta(\xi, \tau)$ quenches (because it cannot ultimately approach zero everywhere). 
For those who prefer a more convincing proof, one can be outlined as follows. The inequality $\theta(\xi, \tau) \geq \theta(\xi, 0)$ implies that $(\partial \theta / \partial \tau)_{\tau=0}>0$. If we consider the function $w=$ $(\partial \theta / \partial \tau) e^{-\gamma \tau}$, we find from Eq. (2.3a) that $\partial w / \partial \tau=\frac{1}{\xi^{2}} \frac{\partial}{\partial \xi}\left(\xi^{2} \frac{\partial w}{\partial \xi}\right)+[\dot{g}(\theta)-\gamma] w=0$, and $w(\xi, 0)>0$. Then, by using a reasoning similar to that used in the appendix, we can show that $w>0$, that is, that $\partial \theta / \partial \tau>0$ for all $\tau$. Thus, $\theta$ always increases everywhere-exactly the meaning of "quench." What we have proved is that any initial condition $\theta(\xi, 0)=(1+|\epsilon|) v(\xi)$ leads to a quench. Quite similarly, we can prove that any initial condition $\theta(\xi, 0)=(1-|\epsilon|) v(\xi)$ recovers.

What does this tell us about the quench energy? Nothing, actually, as long as we keep to the definition of quench energy given in the first sentence of this paper. That definition, based on sudden energy deposition at a point, rests upon the delta-function heat source given in Eq. (2.5), which does not appear in Wilson's work. But Wilson's estimate tells us something about the so-called minimum quench energy (MQE), which is defined a little different'y from what we have defined as the delta-function quench energy.

\section{THE MINIMUM QUENCH ENERGY}

It is possible to deposit large amounts of energy in a magnet without causing a quench. For example, we can heat the conductor everywhere to just below the currentsharing threshold. Thus, there are initial states with formation energies far in excess of the delta-function quench energy that recover. It seems plausible that there is a worst way to distribute the initial heat. The quench energy for this spatial distribution will be a minimum. The quench energy for any other spatial distribution will be higher. Because it is by no means clear that the worst initial heat deposit is a point deposit, all we can say about $q_{\delta}$ is that $q_{\delta}>\mathrm{MQE}$. And because the estimate of Sect. 2 is a lower bound to $q_{\delta}$, it sheds no light at all on the magnitude of the MQE.

Wilson's estimate is also an upper bound to the MQE because any state that quenches must have a formation energy greater than the MQE. Wilson correctly uses the minimum formation energy over $R$ to obtain the lowest upper bound attainable with his method.

One way in which my formula and Wilson's formula are related is that they cannot be simultaneously accurate. The ciagram in Fig. 1 shows the relation between the two estimates. The dotted regions show the domain of admissible values of $q_{\delta}$ and the MQE. The dashed lines illustrate possible locations for $q_{\delta}$ and the MQE (remember that $q_{\delta}>\mathrm{MQE}$ ). If the estimate for $q_{\delta}$ is accurate, the LHS dashed line would have to be close to the value $6 \pi^{2}$, depressing the RHS dashed line by at least a factor of 6 below the estimate of the MQE. Conversely, if the estimate of the MQE is accurate, the RHS dashed line would have to be close to the value 356.4, elevating the LHS dashed line by at least a factor of 6 above the estimate of $q_{j}$.

\section{ANOTHER METHOD OF DETERMINING $q_{\delta}$}

If we compare the solutions $\theta_{1}(\xi, \tau)$ and $\theta_{2}(\xi, \tau)$ of Eq. (2.3a) referring to two different rates of Joule heat production $g_{1}(\theta)>g_{2}(\theta)$ but having the same initial condition as in Eq. (2.5), we find that $\theta_{1}>\theta_{2}$. This is both plausible on physical grounds and provable using the technique described in the appendix. The function $g(\theta)=\theta / i$ majorizes the function $g_{2}(\theta)$ given in Eq. (2.8). With this function, Eq. (2.3a) is directly solvable:

$$
\theta_{1}=q \frac{\exp \left(\tau / i-\xi^{2} / 4 \tau\right)}{(4 \pi \tau)^{3 / 2}}>\theta_{2} \text {. }
$$


The lower limit $6 \pi^{2}$ for $C_{\delta}$ in the 3-D case illuminates an error that can be made in the uncritical application of dimensional analysis: unjustifiably taking undetermined constants to be close to one. There is no reason that this should be so, and in the present example it would lead to estimates of $q_{\delta}$ that are inaccurate by nearly two orders of magnitude.

The exact value of $q_{\delta}$ can be determined by trial and error through repeated numerical solution of the heat balance equation with the source term of Eq. (2.5). But no such brute force approach seems feasible for calculating the MQE. The calculation of $C_{\mathrm{MQE}}$ or even the construction of a nonzero lower bound to it are mathematical problems that still elude solution.

Finally, it should be noted that the simplified form [Eq. (2.8)] of the Joule power function that both Wilson and I use restricts our results to values of $i$ close to one.

\section{APPENDIX}

We wish to show that any function $w$ obeying boundary conditions of Eq. (3b) and satisfying the differential inequality of $\mathrm{Eq}$. (3a) must be positive. The method of proof is reductio ad absurdum, and we begin with the assumption that $w \leqslant 0$ somewhere in the interior of the rectangle shown in Fig. 2 -in the neighborhood of point $P$, for example. No such point can be a minimum because at a minimum, $\partial w / \partial:=\partial w / \partial \xi=0$ and $\partial^{2} w / \partial \xi^{2}>$ 0 . These values, together with $w \leqslant 0$, cannot satisfy the differential inequality of Eq. (3a). Hence, at any interior point at which $w \leqslant 0$, we can find a direction in which $w$ decreases. Following the direction of fastest decrease, we eventually reach the lowest point, which must necessarily lie on the boundary. Since $w=0$ on $O A$ and $A C$, the lowest point, $Q$ (at which $w<0$ ), must lie either on $\mathrm{BC}$ or $\mathrm{OB}$. If it lies on $\mathrm{BC}$, then from $\partial w_{\mathrm{Q}} / \partial \xi=0$, $\partial^{2} w_{\mathrm{Q}} / \partial \xi^{2}>0$, and $w_{\mathrm{Q}}<0$, it follows that $\partial w_{\mathrm{Q}} / \partial \tau>0$. But this means there are inierior points near $Q$ for which $w<w_{Q}$, a contradiction. Similarly, if $Q$ lies on $O B$, then from $\partial w_{\mathrm{Q}} / \partial \tau=\partial w_{\mathrm{Q}} / \partial \xi=0$ and $w_{\mathrm{Q}}<0$, it follows that $\partial^{2} w_{\mathrm{Q}} / \partial \xi^{2}<0$. But this means there are interior points near $Q$ for which $w<w_{Q}$, again a contradiction. Consequently, the assumption that $w \leqslant 0$ anywhere leads to a contradiction and, therefore, $w>0$ everywhere.

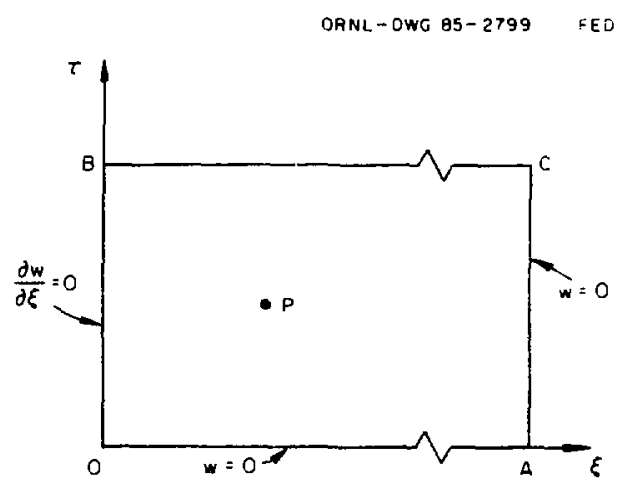

Fig. 2. A rectangle in the $(\xi, \tau)$ plane used for the discussion of the properties of the functions of $w$ obeying the boundary conditions of Eq. (3b) and satisfying the differential inequality of Eq. (3a). The boundary $A C$ is taken sufficiently far to the right that $w$ may be taken to be zero on it. 


\section{REFERENCES}

1. L. Dresner; Quench energies of potted magnets, in "Proc. 27th Meeting of the Japanese Cryogenic Engineering Society," Tokyo (Nov. 4-6, 1981), p. 15.

2. L. Dresner, Quench energies of potted magnets, IEEE Trans. Mag. MAG-21 2: 392-95 (March 1985).

3. M. N. Wilson, "Superconducting Magnets," Clarendon Press, Oxford (1983), pp. 79-84.

4. See, for example, M. H. Protter and H. F. Weinberger, "Maximum Principles in Differential Equations," Prentice-Hall, Englewood Cliffs, New Jersey (1967).

\section{DISCLAIMER}

This report was prepared as an account of work sponsored by an agency of the United States Government. Neither the United States Government nor any agency thereof, nor any of their employees, makes any warranty, express or implied, or assumes any legal liability or responsibility for the accuracy, completeness, or usefulness of any information, apparatui, product, or process disclosed, or represents that its use would not infringe privately owned rights. Reference herein to any specific commercial product, process, or service by trade name, trademark, manufacturer, or otherwise does not necessarily constitute or imply its endorsenient, recommendation, or favoring by the United States Government or any agency thereof. The views and opinions of authors expressed berein do not necessarily state or reiect those of the United States Government or any agency thereof. 